\title{
O ensino do manejo precoce da ansiedade por profissionais de saúde: um relato de
}

\section{experiência}

\author{
Teaching early anxiety management by healthcare professionals: an experience report \\ La enseñanza de la gestión de la ansiedad temprana por profesionales de la salud: un informe de \\ experiencia
}

Recebido: 21/08/2021 | Revisado: 26/08/2021 | Aceito: 29/08/2021 | Publicado: 31/08/2021

\author{
Amanda Sales Cafezeiro \\ ORCID: https://orcid.org/0000-0003-1160-0872 \\ Universidade do Estado da Bahia, Brasil \\ E-mail: amandacafezeiro@hotmail.com \\ Guilia Rivele Souza Fagundes \\ ORCID: https://orcid.org/0000-0003-1834-8278 \\ Universidade do Estado da Bahia, Brasil \\ E-mail: guilia_matina@hotmail.com \\ Carolle Gomes Fernandes Ribeiro \\ ORCID: https://orcid.org/0000-0003-2866-3511 \\ Universidade Estadual do Sudoeste da Bahia, Brasil \\ E-mail: carollegomesf@hotmail.com \\ Murillo Santos Souza \\ ORCID: https://orcid.org/0000-0001-9298-9845 \\ Universidade Estadual do Sudoeste da Bahia, Brasil \\ E-mail: murillo332@ hotmail.com \\ Ana Cristina Santos Duarte \\ ORCID: https://orcid.org/0000-0002-3537-9095 \\ Universidade Estadual do Sudoeste da Bahia, Brasil \\ E-mail: anacristina@uesb.edu.br
}

\begin{abstract}
Resumo
Introdução: o presente relato de experiência é o resultado de uma intervenção em Psicoeducação para o manejo precoce da ansiedade realizada com profissionais de saúde na disciplina Processo de Ensino-Aprendizagem em Ciências da Saúde do Programa de Pós-graduação em Enfermagem e Saúde de uma Universidade Estadual no interior da Bahia. Objetivo: o objetivo da intervenção foi discutir com profissionais de saúde conceitos fundamentais da Psicoeducação em Ansiedade articulados às práticas assistenciais em saúde. Metodologia: Para tanto, utilizou-se como técnica o role playing ou método de interpretação de papéis como metodologia ativa de ensino. Já como método avaliativo foi observada a participação dos profissionais durante a intervenção. Resultados e discussões: Conforme apontado pela teoria, o método do role-playing se mostrou importante metodologia para sensibilização e construção de aprendizagens significativas com os profissionais de saúde acerca do manejo precoce da ansiedade. Conclusão: Como foi possível constatar, o ensino do manejo precoce da ansiedade aos profissionais de saúde por meio de metodologias ativas como o Role playing para a compreensão da importância da psicoeducação se mostrou extremamente eficiente na medida em que possibilitou aos participantes, através da identificação de experiências pessoais com a ansiedade, a compreensão da importância do seu manejo, bem como da capacidade que possuem para tratar do assunto em seus serviços de saúde e demais áreas de atuação.
\end{abstract}

Palavras-chave: Ansiedade; Educação em saúde; Profissionais da saúde.

\begin{abstract}
Introduction: this experience report is the result of an intervention in Psychoeducation for the early management of anxiety carried out with health professionals in theTeaching-Learning Process in Health Sciences discipline of a Postgraduate Program in Nursing and Health of a State University in the interior of Bahia. Objective: the objective of the intervention was to provide health professionals with fundamental concepts of Psychoeducation in Anxiety Articulated With Healthcare Practices. Methodology: For this purpose, role playing or role-playing method was used as an active teaching methodology. As an evaluative method, the participation of professionals during the intervention. Results and discussions: As pointed out by the theory, the role-playing method proved to be an important methodology for raising awareness and building meaningful learning with health professionals about the early management of anxiety.Conclusion: As it was possible to observe, the teaching of early anxiety management to health professionals through active methodologies such as role playing to understand the importance of psychoeducation
\end{abstract}


proved to be extremely efficient in that it enabled the participants, through the identification of personal experiences with anxiety, the understanding of the importance of its management, as well as the capacity they have to deal with the subject in their health services and other areas of expertise.

Keywords: Anxiety; Health education; Health personnel.

\section{Resumen}

Introducción: este relato de experiencia es el resultado de una intervención en Psicoeducación para el manejo temprano de la ansiedad realizada con profesionales de la salud en la disciplina Proceso de Enseñanza-Aprendizaje en Ciencias de la Salud de un Programa de Postgrado en Enfermería y Salud de una Universidad Del Estado en el interior de Bahía. Objetivo: el objetivo de la intervención fue dotar a los profesionales de la salud de conceptos fundamentales de Psicoeducación en Ansiedad articulados con prácticas asistenciales. Metodología: Para ello se utilizó el método role playing o role-playing como metodología de enseñanza activa. Como método evaluativo, se observó la participación de los profesionales durante la intervención. Resultados y discusiones: Como señala la teoría, el método de juego de roles demostró ser una metodología importante para crear conciencia y construir un aprendizaje significativo con los profesionales de la salud sobre el manejo temprano de la ansiedad. Conclusión: Como se pudo observar, la enseñanza del manejo temprano de la ansiedad a los profesionales de la salud a través de metodologías activas como el role playing para comprender la importancia de la psicoeducación resultó ser sumamente eficiente en cuanto permitió a los participantes, a través de la identificación de experiencias personales con ansiedad, la comprensión de la importancia de su gestión, así como la capacidad que tienen para abordar el tema en sus servicios de salud y otras áreas de especialización.

Palabras clave: Ansiedad; Educación para la salud; Personal de salud.

\section{Introdução}

O termo ansiedade refere-se à uma resposta natural do organismo para o enfrentamento de situações que lhe indiquem perigo (Scherer, 2020), configurando-se como um comportamento adaptativo que o prepara para a luta ou a fuga (Sadock, Sadock \& Ruiz, 2017). Nestes termos, seu papel é fundamental à preservação da vida, atuando de forma positiva, inclusive, no aumento da atenção e desempenho de tarefas.

O sentimento de expectativa para a realização de determinadas atividades como uma prova, entrevista de emprego ou a apresentação de um espetáculo pode estimular o indivíduo a se preparar melhor, o que pode favorecer seus resultados e, assim, a ansiedade, se controlada, pode ser entendida como favorável e necessária, não demonstrando diminuição da qualidade de vida das pessoas e desaparecendo tão logo o perigo se encerra.

A literatura especializada caracteriza a ansiedade por um estado desagradável e difuso de apreensão, que frequentemente cursa com palpitações, desconforto abdominal, aperto no peito e cefaleia (Kaplan, Sadock \& Grebb, 1997).

Há situações, todavia, em que o quadro de ansiedade vivenciado pelo indivíduo é tão intenso que passa a comprometer sua qualidade de vida, afetando negativamente seu funcionamento e gerando sofrimento. Sendo assim, compreende-se que a ansiedade se tornou patológica, caracterizando um transtorno de ansiedade.

Para Araújo (2011) a ansiedade passa a ser prejudicial ao organismo no momento em que o deixa em constante estado de alerta. Neste caso, como afirma Townsend (2011), os indivíduos começam a apresentar reações amplificadas e, geralmente, incompatíveis com sua situação disparadora, fato que em níveis extremos tem o potencial de incapacitar seu portador.

De acordo com a $5^{\text {a }}$ edição do Manual Diagnóstico e Estatísticos de Transtornos Mentais (DSM-5), da Associação Americana de Psiquiatria, os Transtornos de Ansiedade consistem em uma ampla categoria de transtorno com aspectos em comum, tais como medo, apreensão, ansiedade excessiva que causam perturbações comportamentais. Seus sintomas costumam ser classificados em dois grandes grupos: subjetivos e físicos. Os de natureza física frequentemente se apresentam como palpitação, falta de ar, aperto no peito, sensação de bolo na garganta, transpiração excessiva, formigamento e náuseas. Já os sintomas subjetivos são descritos como angústia, preocupação excessiva, excesso de pensamentos, medo e inquietações.

Os comprometimentos causados pela ansiedade também possuem forte impacto nos processos de aprendizagem, pois habitualmente interferem no pensamento, atenção, memória e na percepção. A literatura descreve confusões perceptivas que 
alteram a percepção de tempo e espaço e dos significados dos acontecimentos, o que pode perturbar, inclusive, a capacidade de seu portador de se relacionar (Sadock, 2017).

Como é possível notar, o transtorno de ansiedade compromete amplamente as distintas áreas da vida de discentes, além de estar cada vez mais evidente, o que colabora para a utilização imprópria de fármacos o que, consequentemente, pode acarretar outros problemas (Costa et al.,2017).

Dalgalarrondo (2019) descreve sintomas importantes a serem identificados em um processo diagnóstico do Transtorno de Ansiedade: dificuldade para relaxar, insônia, problemas de concentração, maior irritabilidade e sentimentos de angústia. Frequentemente, estes sintomas cursam com outros de ordem física: dores de cabeça e musculares, problemas gastrointestinais, sudorese, sensação de coração disparado, tontura, dentre outros.

Estes sintomas não são novos conhecidos dos profissionais de saúde e até mesmo da população, muito pelo contrário, sempre fizeram parte do cotidiano das pessoas, especialmente com as transformações sociais do mundo contemporâneo, que estimulam o excesso de produtividade, a competitividade, a substituição compulsiva dos bens de consumo, ou seja, de um mundo cada vez mais exigente, responsável pela intensificação de transtornos como o da Ansiedade (Guimarães, 2015).

Andrade (2019) apresenta dados que chamam a atenção. Segundo o autor, $1 / 3$ da população chega a ser afetada por algum distúrbio de ansiedade durante a vida, o que lhes gera diversos comprometimentos físicos, laborais, relacionais e intensifica a demanda por cuidados em saúde, ocasionando grande impacto econômico para a sociedade.

São grandes os índices que revelam a presença de ansiedade em números expressivos de usuários do sistema de saúde. O quadro costuma se apresentar de modo diverso variando desde uma patologias de base, comorbidades com outras patologias e efeitos secundários de substâncias e medicamentos (Pinheiro, 2019). Perante as limitações funcionais e os malefícios associados à ansiedade, torna-se crucial aumentar o alcance da prestação de cuidados por parte dos profissionais de saúde para seu diagnóstico e manejo precoces, como nos afirma de Melo, 2016, p.01:

Uma das maiores barreiras para o desenvolvimento de propostas terapêuticas mais eficazes em saúde mental nos serviços de saúde nos países em desenvolvimento tem sido o número insuficiente de profissionais devidamente capacitados e supervisionados para oferecerem cuidados de saúde mental. A qualificação em saúde mental muitas vezes tem sido negligenciada por parte do poder público e também pelos profissionais de enfermagem, o que contribui para a assistência pouco especializada, inadequada e de baixa qualidade nos serviços de atenção primária à saúde. Essa deficiência de incorporação de conhecimentos qualificados por parte dos profissionais pode estar associada a atitudes desfavoráveis a uma assistência de qualidade (de Melo, 2016, p 01).

Sendo assim, nota-se a importância de que os profissionais adquiram competências para reconhecer quadros de ansiedade, como também para poder intervir na redução dos mesmos através de intervenções não-farmacológicas e precoces (Pinheiro, 2019).

As intervenções psicoeducativas, neste sentido, que visam reduzir a ansiedade na pessoa, têm sido recursos valiosos ao alcance de todos os profissionais de saúde, que precisam compreender a eficiência das práticas não-farmacológicas para o sucesso da terapêutica.

A psicoeducação é definida como o emprego de uma técnica psicoterapêutica, utilizada como parte do tratamento de transtornos psiquiátricos, se tratando do fornecimento do máximo possível de informações referentes ao quadro clínico do paciente (Justo \& Lipp, 2015; Tomita et al., 2018).

Para Fenwick et al., 2018 a psicoeducação busca investir no modelo educativo como parte do tratamento, já que concebe o indivíduo em sua integralidade, baseado na perspectiva biopsicossocial. A técnica busca, através do fornecimento de informações que auxiliem o paciente no manejo de seu quadro, estimular mudanças de comportamento e emocionais por gerar modificações no funcionamento cognitivo. 
Reconhecendo o valor social e a necessidade da melhoria na qualidade de vida das pessoas por meio da qualificação dos profissionais de saúde no trato com este problema de grande impacto, busca-se através deste artigo realizar um relato de experiência no qual são descritos, analisados e avaliados os resultados de uma intervenção em Psicoeducação para o manejo precoce da ansiedade, realizada com profissionais de saúde na disciplina Processo de Ensino-Aprendizagem em Ciências da Saúde de um Programa de Pós-graduação em Enfermagem e Saúde de uma Universidade Estadual no interior da Bahia, objetivando discutir conceitos fundamentais da Psicoeducação em Ansiedade articulados às práticas assistenciais em saúde.

\section{Metodologia}

Trata-se de um relato de experiência que conforme Daltro e Farias (2019, p224), "permite a criação de narrativa científica, especialmente no campo das pesquisas capazes de englobar processos e produções subjetivas", da estratégia de aprendizagem adotada na disciplina Processo de Ensino Aprendizagem em Ciências da Saúde, ofertada no Mestrado de Ciências da Saúde do Programa de Pós-Graduação em Enfermagem e Saúde de uma universidade do interior da Bahia, com carga horária de 30 horas, no semestre de 2021.1. O grupo de discentes era composto por 12 profissionais de saúde; dos quais 6 eram enfermeiros, 2 profissionais de educação física, 1 profissional com formação em enfermagem e educação física, 2 cirurgiãs dentistas e 1 psicóloga.

Utilizou-se o role playing (método de interpretação de papéis) como metodologia ativa de ensino. Este possibilita que uma pessoa assuma o papel de outra com o propósito de sensibilizar os demais. A utilização da dramatização torna a vivência mais próxima do real, sendo considerada uma prática de ensino de simulação ao proporcionar a imersão e a experiência vivida. A aprendizagem proporcionada não é somente individual como também coletiva (Riera, Cibanal \& Mora, 2016).

Foi realizado um programa de auditório através da plataforma Google Meet, denominado "Bem-estar na saúde", apresentado por uma Enfermeira, entrevistando profissionais da saúde sobre o tema ansiedade.

Na primeira parte da dinâmica, a entrevistadora direcionou perguntas acerca da temática aos especialistas, em seguida foi aberto um momento para perguntas feitas pelos internautas do programa, que eram, na verdade, os discentes da disciplina. As perguntas foram elaboradas previamente pelos próprios alunos e apareciam na tela do programa acompanhadas das fotos dos mesmos, o que foi motivo de entusiasmo para a turma. As perguntas eram relacionadas à ansiedade, porém especificamente no contexto de trabalho de cada um desses profissionais. Então, enfermeiros que trabalhavam em UTIs fizeram perguntas sobre a ansiedade neste contexto, profissionais que trabalhavam na atenção básica de saúde da mesma forma, também ocorreram questionamentos sobre a ansiedade e aspectos epidemiológicos.

No terceiro momento houve a participação da Cirurgiã-Dentista, que relatou sobre a sua experiência com pacientes ansiosos na prática clínica, apresentando a técnica do Dizer-Mostrar-Fazer como estratégia para o manejo precoce da ansiedade nos pacientes.

Esta é uma técnica utilizada por muitos profissionais de Odontopediatria para moldar o comportamento da criança, sendo amplamente aceita por crianças, pais e profissionais. A técnica envolve explicações dos procedimentos no nível que a pessoa possa compreender, antecipando ao paciente por meio da fala, imagens, aparelhos ou recursos lúdicos tudo que acontecerá no atendimento (Massara \& Redua, 2013). É um momento no qual o profissional deve estimular que a pessoa fale e tire as suas dúvidas, podendo ser adaptada por todos os profissionais de saúde para a redução da ansiedade em seus pacientes.

No quarto estágio da dinâmica, a entrevistadora abriu espaço para a interação com o auditório do programa, representado pelos discentes. Nesta ocasião, era possível que eles relatassem, por meio da interpretação de personagens experiências pessoais de ansiedade, fato que trouxe muita riqueza ao momento, pois havia liberdade para falar de si por meio de um personagem, mobilizando aspectos afetivos dos aprendizes. 
Para o fechamento da dinâmica, a entrevistadora convidou o profissional de educação física para realizar uma atividade de ginástica laboral aplicada a ergonomia, dando dicas para que os discentes, interpretando a plateia do programa, executassem sob sua supervisão. Esta atividade tinha como finalidade a utilização da ginástica laboral para a redução da ansiedade.

Por se tratar de uma atividade educativa, foi realizada, ao final da dinâmica, uma avaliação da intervenção por meio da aplicação de questionário semi-estruturado na plataforma Google Forms, contendo três perguntas que versavam sobre o conteúdo teórico apresentado e sobre suas impressões pessoais da dinâmica.

\section{Resultados e Discussão}

O componente curricular Processo de Ensino-Aprendizagem em Ciências da Saúde, ministrado no Programa de PósGraduação em Enfermagem e Saúde na Universidade do Sudoeste da Bahia- UESB em Jequié - BA, possibilitou aos mestrandos o desenvolvimento de uma oficina acerca da Ansiedade; tema extremamente atual principalmente em tempos de pandemia do Covid -19 .

Foi possível observar que a oficina permitiu que os discentes se auto- avaliassem e identificassem situações ou vivências pessoais que poderiam estar associadas a quadros de ansiedade. O relato abaixo evidencia a visão da participante acerca da temática.

“... estamos vivenciando um momento de pandemia e gerenciar tudo isso tem sido bem difícil, devido aos momentos de incertezas, de dificuldade global e aí, vem questões financeiras que envolvem isso e também questões de família, situações na sua própria comunidade de amigos, falecimento de pessoas amigas, e isso tudo dificulta muito como a gente lidar né? com a situação de sobrecarga de trabalho, que antes lidava com uma certa facilidade, isso tudo, provocou em mim um quadro de ansiedade, realmente muito forte tanto na parte psicológica e emocional, na mental quanto no físico. No momento, estou buscando ajuda profissional e precisando também eliminar alguma atividade da minha rotina pra conseguir dar continuidade para ter um pouco de qualidade de vida...”. (Participante 1).

Esta fala expressa claramente o impacto que a técnica do Role Playing pode ter por suscitar as experiências pessoais dos indivíduos, achado que corrobora com o pensamento de Riera, Cibanal e Mora (2016) ao afirmarem que a dramatização sensibiliza e propicia uma imersão na experiência. Esse apelo afetivo, que propicia reflexões íntimas, é um fator propulsor para o aprendizado, por isso a relevância da aplicação da técnica.

Pode-se igualmente identificar no depoimento os efeitos da denominada sociedade da ansiedade, cujo excesso de demandas, conforme afirmado por Guimarães (2015), oriundas da sobrecarga de tarefas e exigências competitivas, geram forte impacto emocional e causam transtornos inesperados.

A ansiedade apresenta inicialmente de maneira branda, até que seus sinais e sintomas começam a intensificar- se, por vezes, pode não ser tão percebida pelos indivíduos acometidos pelo transtorno, o que consequentemente colabora para a redução na capacidade de adaptação. Dessa maneira, a pessoa com ansiedade não acredita nos riscos, não se dando conta de encarar as intimidações acerca das suas condições físicas e psicológicas (Santos \& Simões, 2014).

Estudo demonstra que o transtorno de ansiedade entre profissionais pode estar ligado a distintos fatores como insônia, se sentir pressionado no local de atuação, bem como, ter realizado algum tipo de tratamento para o transtorno no último ano (Moura et al.,2018).

Durante as explanações os profissionais de saúde ressaltaram sobre as manifestações clínicas comuns presentes em diversos quadros de ansiedade.

“... Então, eu desenvolvi vários sinais físicos, sudorese intensa, palpitações, perda de memória, déficit de atenção, e 
por fim foi somatizando ao meu organismo e eu precisei de atendimento clínico para também mensurar e enquanto isso na minha parte emocional tava sendo afetada, eu vou precisar fazer um tratamento e a gente precisar reavaliar pra identificar até que ponto essa ansiedade está sendo benéfica e que ponto começa a se prejudicar”. (Participante 1).

Os achados da literatura descrevem o quadro sintomatológico narrado. Sadock (2017), por exemplo, aborda os prejuízos cognitivos causados pela ansiedade que alteram negativamente a memória e a aprendizagem, bem como os variados sinais e sintomas físicos descritos por Kaplan, Sadock e Grebb (1997) e Dalgalarrondo (2019.)

Inúmeras vezes, para evitar o agravamento do quadro clínico, faz - se necessário o uso de ferramentas auxiliadoras no tratamento do transtorno de ansiedade como a realização de atividade física, meditação, métodos não-farmacológicos, conforme defendido por Pinheiro (2019) e em alguns casos, é necessário o uso de medicamentos, como relatado pelo Participante 2:

"Manejo a ansiedade com ansiolítico, mas a atividade física tem um papel fundamental". (Participante 2).

Os profissionais de saúde devido à sobrecarga de trabalho, rotinas estressantes e exaustivas, são inúmeras vezes acometidos pelo Transtorno de Ansiedade, o que ficou claro durante a realização do evento por meio das falas dos participantes que demonstraram identificação e autoanálise a partir do conteúdo apresentado. Este aspecto pode ter favorecido a participação dos discentes. Os próprios relataram que a metodologia utilizada para realizar a abordagem de uma temática tão complexa, colaborou para que a abordagem acontecesse de forma mais clara, leve, precisa e objetiva.

"é um tema tão interessante, está na pauta do dia, inclusive faz parte da nossa realidade aqui enquanto trabalhadores da saúde e parabenizar até mesmo a maneira que conduziram nos deu também ferramentas para lidar melhor com essa ansiedade e realmente com esse programa de hoje eu consigo perceber que nós somos seres complexos, múltiplos, temos coisas que nos favorece e que nos desfavorece". (Participante 3).

"o programa foi uma estratégia bem utilizada, em nenhum momento pareceu que eu estava assistindo uma aula". (Participante 4).

Nota-se que as intervenções psicoeducativas, pelo caráter informativo e neste caso vivencial, podem cumprir uma importante função no que tange ao estímulo à auto identificação de sintomas de ansiedade e do mesmo modo que contribuíram com os profissionais também podem servir como intervenções não farmacológicas nos diversos serviços de saúde.

\section{Conclusão}

Como foi possível constatar, o ensino do manejo precoce da ansiedade aos profissionais de saúde por meio de metodologias ativas como o Role playing para a compreensão da importância da psicoeducação se mostrou extremamente eficiente na medida em que possibilitou aos participantes, através da identificação de experiências pessoais com a ansiedade, a compreensão da importância do seu manejo, bem como da capacidade que possuem para tratar do assunto em seus serviços de saúde e demais áreas de atuação.

Notou-se também que a oficina permitiu a ampliação do conhecimento acerca da temática em estudo, refletir sobre as diversas possibilidades de uso da psicoeducação, além de estimular a produção de novos estudos com o intuito de demonstrar a real necessidade de discussões que envolvam o tema, principalmente no que menciona a prevenção.

Salienta -se que os gestores de saúde, dada a relevância do problema da ansiedade, devem estimular a realização de encontros com esta temática, já que é um transtorno muito evidente e vivenciado tanto por profissionais de saúde, quanto por seus pacientes, podendo ser considerado como um problema de saúde pública. 
Research, Society and Development, v. 10, n. 11, e265101119673, 2021

(CC BY 4.0) | ISSN 2525-3409 | DOI: http://dx.doi.org/10.33448/rsd-v10i11.19673

\section{Referências}

Andrade, J. V., Pereira, L. P., Vieira, P. A., da Silva, J. V. S., Silva, A. de M., \& Bonisson, M. B. (2019). Ansiedade, um dos problemas do século XXI. Rev de Saúde da ReAGES. 4( 2), 34-39.

Araújo, N. G. (2011). Fobia específica: passo a passo de uma intervenção bemsucedida. Revista Brasileira de Terapia Cognitiva, 7 (2), $37-45$.

Associação Americana de Psiquiatria (2014). Manual Diagnóstico e Estatísticos de Transtornos Mentais (5 a ed. )

Dalgalarrondo, P. (2019). Psicopatologia e Semiologia dos transtornos mentais. Artmed.

Daltro, M. R., \& de Faria, A. A. (2019). Relato de experiência: Uma narrativa científica na pós-modernidade. Estudos e pesquisas em psicologia, 19(1), 223237.

da Silva Maia, R., de Araújo, T. C. S., \& Maia, E. M. C. (2018). Aplicação da Psicoeducação na Saúde: revisão integrativa. Revista Brasileira de Psicoterapia, 20(2), 53-63.

De Melo, Z. M., Pegoraro, N. P. J., dos Santos, M. A., \& Pillon, S. C. (2016) Atitudes e conhecimentos de técnicos de enfermagem sobre cuidados a pacientes com transtornos mentais. Rev. Eletr. Enf. 18. 1141.

de Oliveira, K. M. A., Marques, T. C. \& da Silva, C. D. A. (2020). Cuidados de enfermagem frente ao transtorno de ansiedade. Hígia-Revista De Ciências Da Saúde E Sociais Aplicadas Do Oeste Baiano, 5(1).

Guimarães, A. M. V., da Silva Neto, A. C., Vilar, A. T. S., da Costa Almeida, B. G., de Oliveira Fermoseli, A. F., \& de Albuquerque, C. M. F. (2015). Transtornos de ansiedade: um estudo de prevalência sobre as fobias específicas e a importância da ajuda psicológica. Caderno de Graduação-Ciências Biológicas e da Saúde-UNIT-ALAGOAS, 3(1), 115-128.

Fenwick, J., Toohill, J., Slavin, V., Creedy, D. K., \& Gamble, J. Improving psychoeducation for women fearful of childbirth: Evaluation of a research translation project. Women Birth [periódico na Internet].2018[citado 2018 Mar02];31(1):1-9.

Justo, A. P., Matos, E. G., \& Lipp, M. E. Cognitive-behavioral therapy of a case ofobsessive-compulsive disorder. Rev. Bras. Ter. Cogn. [periódico na Internet]. 2015 Jun[citado 2018 Fev 28]; 11(1): 10-24. https:// dx.doi.org/10.5935/1808-5687.20150003.

Kaplan, H., Sadock, B., \& Grebb, J. (1997) - Compêndio de Psiquiatria: Ciências do comportamento e psiquiatria clínica. (7 Ed.) Porto Alegre: Artmed.

Massara, M. L. A., \& Redua, P. C. B. (2013). Manual de referência para procedimentos clínicos em Odontopediatria. (2 ${ }^{\text {a }}$ Ed.): Santos. p. 30

Moura, A., Lunardi, R., Volpato, R., Nascimento, V., Bassos, T., \& Lemes, A. (2018). Fatores associados à ansiedade entre profissionais da atenção básica. Revista Portuguesa de Enfermagem de Saúde Mental, (19), 17-26.

Pinheiro, A. L. D. P. (2019). Intervenções não-farmacológicas na redução da ansiedade em enfermagem de saúde mental (Doctoraldissertation).

Riera, J. R. M., Cibanal, J. L., \& Mora, M. J. P. (2010). Using role playing in theintegration of knowledge in theteaching-learning process in nursing: assessment of students. Contexto Enferm, 19(4),618-626.

Sadock, B. J., Sadock, V. A., \& Ruiz, P. (2017). Compêndio de Psiquiatria. Ciência do Comportamento e Psiquiatria Clínica. Artmed

Scherer, S. G. (2020). A eficácia da auriculoterapia nos transtornos de ansiedade: uma revisão sistemática. Naturologia-Pedra Branca.

Townsend, M. C. (2011). Enfermagem em Saúde Mental e Psiquiátrica: Conceitos de Cuidado na Prática Baseada na Evidência (6 ed.). Loures: Lusociência.

Tomita, T., Kudo, S., Sugawara, N., Fujii, A., Tsuruga, K., Sato, Y., et al. Timing of psychoeducation for patients with depression Who were treated with antidepressants: When should patients receive psychoeducation. Neuropsychiatr Dis Treat [periódico na Internet]. 2018 [citado 2018 Mar02]; 14:505-510. 